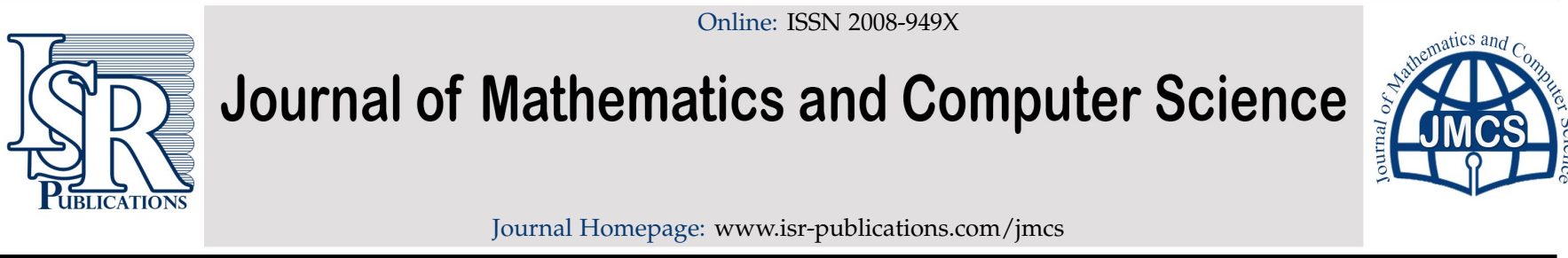

\title{
Permanence of a nonlinear mutualism model with time vary- ing delay
}

Runxin Wu

Mathematics and Physics Institute, Fujian University of Technology, Fuzhou, Fujian, 350014, P. R. China.

\section{Abstract}

Sufficient conditions are obtained for the permanence of the following nonlinear mutualism model with time varying delay

$$
\begin{aligned}
& \frac{d N_{1}(t)}{d t}=r_{1}(t) N_{1}(t)\left[\frac{K_{1}(t)+\alpha_{1}(t) N_{2}^{\beta_{1}}\left(t-\tau_{2}(t)\right)}{1+N_{2}^{\beta_{1}}\left(t-\tau_{2}(t)\right)}-N_{1}^{\delta_{1}}\left(t-\sigma_{1}(t)\right)\right], \\
& \frac{d N_{2}(t)}{d t}=r_{2}(t) N_{2}(t)\left[\frac{K_{2}(t)+\alpha_{2}(t) N_{1}^{\beta_{2}}\left(t-\tau_{1}(t)\right)}{1+N_{1}^{\beta_{2}}\left(t-\tau_{1}(t)\right.}-N_{2}^{\delta_{2}}\left(t-\sigma_{2}(t)\right)\right],
\end{aligned}
$$

where $r_{i}, K_{i}, \alpha_{i}, \tau_{i}$, and $\sigma_{i}, i=1,2$ are continuous functions bounded above and below by positive constants, $\alpha_{i}>K_{i}, i=1,2$, and $\beta_{i}, \delta_{i}, i=1,2$ are all positive constants.

Keywords: Mutualism, nonlinear, delay, permanence.

2010 MSC: 34C25, 92D25, 34D20.

\section{Introduction}

Throughout this paper, for a continuous function $g(t)$, we set

$$
g^{l}=\inf _{t \in R} g(t), g^{u}=\sup _{t \in R} g(t) .
$$

The aim of this paper is to investigate the persistent property of the following nonlinear mutualism model with time varying delay

$$
\begin{aligned}
& \frac{d N_{1}(t)}{d t}=r_{1}(t) N_{1}(t)\left[\frac{K_{1}(t)+\alpha_{1}(t) N_{2}^{\beta_{1}}\left(t-\tau_{2}(t)\right)}{1+N_{2}^{\beta_{1}}\left(t-\tau_{2}(t)\right)}-N_{1}^{\delta_{1}}\left(t-\sigma_{1}(t)\right)\right], \\
& \frac{d N_{2}(t)}{d t}=r_{2}(t) N_{2}(t)\left[\frac{K_{2}(t)+\alpha_{2}(t) N_{1}^{\beta_{2}}\left(t-\tau_{1}(t)\right)}{1+N_{1}^{\beta_{2}}\left(t-\tau_{1}(t)\right.}-N_{2}^{\delta_{2}}\left(t-\sigma_{2}(t)\right)\right] .
\end{aligned}
$$

We assume that the coefficients of system (1.1) satisfies:

Email address: runxinwu@163.com (Runxin $\mathrm{Wu})$

doi: $10.22436 /$ jmcs.019.02.07

Received: 2019-01-26 Revised: 2019-01-29 Accepted: 2019-04-30 
(A) $r_{i}, K_{i}, \alpha_{i}, \tau_{i}$ and $\sigma_{i}, i=1,2$ are continuous functions bounded above and below by positive constants, $\alpha_{i}>K_{i}, i=1,2$, and $\beta_{i}, \delta_{i}, i=1,2$ are all positive constants.

Let $\tau=\sup _{t}\left\{\tau_{i}(t), \sigma_{i}(t), i=1,2\right\}$, we consider (1.1) together with the following initial conditions

$$
\mathrm{N}_{\mathfrak{i}}(\mathrm{s})=\varphi_{\mathrm{i}}(\mathrm{s}) \geqslant 0, \mathrm{~s} \in[-\tau, 0], \varphi_{\mathfrak{i}}(0)>0 .
$$

It is not difficult to see that solutions of (1.1)-(1.2) are well defined for all $t \geqslant 0$ and satisfy

$$
N_{i}(t)>0 \text { for } t \in R, i=1,2 .
$$

During the past decades, many scholars focused their attention to the study of the dynamic behaviors of the cooperative system, see [1-37]. Stimulated by the work of Gopalsamy [14], Dean [13], Boucher [1], Wolin and Lawlor [1], $\mathrm{Li}$ [21], and $\mathrm{Li}$ and $\mathrm{Xu}$ [24] studied the following two species mutualism model

$$
\begin{aligned}
& \frac{d N_{1}(t)}{d t}=r_{1}(t) N_{1}(t)\left[\frac{K_{1}(t)+\alpha_{1}(t) N_{2}\left(t-\tau_{2}(t)\right)}{1+N_{2}\left(t-\tau_{2}(t)\right)}-N_{1}\left(t-\sigma_{1}(t)\right)\right], \\
& \frac{d N_{2}(t)}{d t}=r_{2}(t) N_{2}(t)\left[\frac{K_{2}(t)+\alpha_{2}(t) N_{1}\left(t-\tau_{1}(t)\right)}{1+N_{1}\left(t-\tau_{1}(t)\right.}-N_{2}\left(t-\sigma_{2}(t)\right)\right] .
\end{aligned}
$$

Under the assumption $r_{i}, K_{i}, \alpha_{i}$ and $\tau_{i}, \sigma_{i}, i=1,2$ are continuous periodic functions with common period $\omega . \alpha_{i}>K_{i}, i=1,2$. By applying the coincidence degree theory, they showed that system (1.3) admits at least one positive $\omega$-periodic solution. After that, by constructing some suitable Lyapunov functional, they also obtained a set of sufficient conditions which ensure the global attractivity of the positive periodic solution. After the works of $[1,13,14,21,24,27,28]$, many scholars $([4,7,8,12,16,23,31,32,34-36])$ done works on this direction. For example, Chen [12] further incorporated the feedback control variables to the system (1.3) and investigated the persistent property of the system. Chen and Xie [8] showed that feedback control variables has no influence to the persistent property of a discrete mutualism model.

It bring to our attention that the model (1.3) is based on the following single species Logistic model:

$$
\frac{d N(t)}{d t}=r(t) N(t)[K(t)-N(t)]
$$

Already, during the past decades, in their series papers, based on the traditional single species Ayala model, Chen and his coauthors $([2,3,5,6,10,11,18-20,22,33])$ proposed several kind of nonlinear population models, and investigated the extinction, persistent, and stability property of the system. For example, Chen [11] investigated the permanence and extinction of following general nonautonomous n-species Gilpin-Ayala competition system

$$
\dot{x}_{i}(t)=x_{i}(t)\left[b_{i}(t)-\sum_{j=1}^{n} a_{i j}(t)\left(x_{j}(t)\right)^{\alpha_{i j}}\right], i=1,2, \ldots, n,
$$

where $b_{i}(t), 1 \leqslant i \leqslant n$ and $a_{i j}(t), i, j=1,2, \ldots, n$ are continuous for $c \leqslant t<+\infty, \alpha_{i j}$ are positive constants. In [10], Chen et al investigated the extinction property of the following two species competitive model:

$$
\begin{aligned}
& \dot{x}_{1}(t)=x_{1}(t)\left[r_{1}(t)-a_{1}(t) x_{1}^{\alpha_{1}}(t)-b_{1}(t) x_{2}^{\alpha_{2}}(t)-c_{1}(t) x_{1}^{\alpha_{1}}(t) x_{2}^{\alpha_{2}}(t)\right], \\
& \dot{x}_{2}(t)=x_{2}(t)\left[r_{2}(t)-a_{2}(t) x_{1}^{\alpha_{1}}(t)-b_{2}(t) x_{2}^{\alpha_{2}}(t)-c_{2}(t) x_{1}^{\alpha_{1}}(t) x_{2}^{\alpha_{2}}(t)\right] .
\end{aligned}
$$

In [6], Chen and Wu studied the persistent and global stability property of the following $n$-species discrete Gilpin-Ayala competition model:

$$
x_{i}(k+1)=x_{i}(k) \exp \left[b_{i}(k)-\sum_{j=1}^{n} a_{i j}(k)\left(x_{j}(k)\right)^{\theta_{i j}}\right],
$$


where $i=1,2, \ldots, n ; x_{i}(k)$ is the density of competition species $i$ at $k$-th generation.

Though much progress has been obtained on the Gilpin-Ayala type system $([2,3,5,6,9-11,15,17-$ $20,22,33,37])$, all of those works are focus on the competition system or predator-prey system, and none of them consider the mutualism model, this motivated us to propose the system (1.1), which is a generalization of system (1.3). Also, as far as population model concerned, the persistent property is one of the most important property of the system, since it represents the long time existence of the species.

The aim of this paper is, by further developing the analysis technique of [2] and the differential inequality theory, to obtain a set of sufficient conditions to ensure the permanence of the system (1.1). More precisely, we will prove the following result.

Theorem 1.1. Under the assumption (A), system (1.1) is permanent, that is, there exist positive constants $m_{i}, M_{i}$, $i=1,2$ which are independent of the solutions of system (1.1), such that for any positive solution $\left(x_{1}(t), x_{2}(t)\right)^{\top}$ of system (1.1) with initial condition (1.2), one has:

$$
m_{i} \leqslant \liminf _{t \rightarrow+\infty} x_{i}(t) \leqslant \limsup _{t \rightarrow+\infty} x_{i}(t) \leqslant M_{i}, i=1,2 .
$$

\section{Proof of the main result}

Now let's state the following lemma which will be useful in the proving of main result.

Lemma 2.1 ([10]). If $\mathrm{a}>0, \mathrm{~b}>0$ and $\dot{x} \geqslant x\left(\mathrm{~b}-\mathrm{ax}{ }^{\alpha}\right)$, where $\alpha$ is a positive constant, when $\mathrm{t} \geqslant 0$ and $x(0)>0$, we have

$$
\liminf _{t \rightarrow+\infty} x(t) \geqslant\left(\frac{b}{a}\right)^{1 / \alpha}
$$

If $\mathrm{a}>0, \mathrm{~b}>0$ and $\dot{\mathrm{x}} \leqslant \mathrm{x}\left(\mathrm{b}-\mathrm{ax} \mathrm{x}^{\alpha}\right)$, where $\alpha$ is a positive constant, when $\mathrm{t} \geqslant 0$ and $\mathrm{x}(0)>0$, we have

$$
\limsup _{t \rightarrow+\infty} x(t) \leqslant\left(\frac{b}{a}\right)^{1 / \alpha}
$$

Now we are in the position of proving the main result of this paper.

Proof of Theorem 1.1. Set

$$
\tau=\sup _{t}\left\{\tau_{i}(t), \sigma_{i}(t), i=1,2\right\}
$$

Let $\left(N_{1}(t), N_{2}(t)\right)$ be any positive solution of system (1.1) with initial condition (1.2). From the first equation of system (1.1) and $\alpha_{1}(t)>K_{1}(t)$ it follows that

$$
\begin{aligned}
\frac{d N_{1}(t)}{d t} & =r_{1}(t) N_{1}(t)\left[\frac{K_{1}(t)+\alpha_{1}(t) N_{2}^{\beta_{1}}\left(t-\tau_{2}(t)\right)}{1+N_{2}^{\beta_{1}}\left(t-\tau_{2}(t)\right)}-N_{1}^{\delta_{1}}\left(t-\sigma_{1}(t)\right)\right] \\
& \leqslant r_{1}(t) N_{1}(t)\left[\frac{\alpha_{1}(t)+\alpha_{1}(t) N_{2}^{\beta_{1}}\left(t-\tau_{2}(t)\right)}{1+N_{2}^{\beta_{1}}\left(t-\tau_{2}(t)\right)}-N_{1}^{\delta_{1}}\left(t-\sigma_{1}(t)\right)\right] \\
& \leqslant r_{1}(t) N_{1}(t) \alpha_{1}(t) \leqslant r_{1}^{u} \alpha_{1}^{u} N_{1}(t),
\end{aligned}
$$

Integrating both sides of (2.1) from $t-\sigma_{1}(t)$ to $t$ leads to

$$
\ln \frac{N_{1}(t)}{N_{1}\left(t-\sigma_{1}(t)\right)} \leqslant \int_{t-\sigma_{1}(t)}^{t} r_{1}^{u} \alpha_{1}^{u} d s \leqslant r_{1}^{u} \alpha_{1}^{u} \tau,
$$

and so

$$
N_{1}\left(t-\sigma_{1}(t)\right) \geqslant N_{1}(t) \exp \left\{-r_{1}^{u} \alpha_{1}^{u} \tau\right\}
$$


Substituting (2.2) into the first equation of system (1.1), it follows that

$$
\begin{aligned}
\frac{d N_{1}(t)}{d t} & \leqslant r_{1}(t) N_{1}(t)\left[\alpha_{1}(t)-N_{1}^{\delta_{1}}\left(t-\sigma_{1}(t)\right)\right] \\
& \leqslant N_{1}(t)\left[r_{1}^{u} \alpha_{1}^{u}-r_{1}^{l}\left(N_{1}(t) \exp \left\{-r_{1}^{u} \alpha_{1}^{u} \tau\right\}\right)^{\delta_{1}}\right] \\
& \left.=N_{1}(t)\left[r_{1}^{u} \alpha_{1}^{u}-r_{1}^{l} N_{1}^{\delta_{1}}(t) \exp \left\{-\delta_{1} r_{1}^{u} \alpha_{1}^{u} \tau\right\}\right)\right] .
\end{aligned}
$$

Thus, as a direct corollary of Lemma 2.1, according to (2.3), one has

$$
\limsup _{\mathrm{t} \rightarrow+\infty} \mathrm{N}_{1}(\mathrm{t}) \leqslant\left(\frac{\mathrm{r}_{1}^{\mathrm{u}} \alpha_{1}^{\mathfrak{u}}}{r_{1}^{\mathrm{l}}} \exp \left\{\delta_{1} r_{1}^{\mathrm{u}} \alpha_{1}^{\mathrm{u}} \tau\right\}\right)^{\frac{1}{\delta_{1}}}=\left(\frac{\mathrm{r}_{1}^{\mathrm{u}} \alpha_{1}^{\mathfrak{u}}}{r_{1}^{\mathrm{l}}}\right)^{\frac{1}{\delta_{1}}} \exp \left\{r_{1}^{\mathrm{u}} \alpha_{1}^{\mathfrak{u}} \tau\right\} \stackrel{\text { def }}{=} M_{1} .
$$

By using the second equation of system (1.1), similarly to the analysis of (2.1)-(2.4), we can obtain

$$
\limsup _{t \rightarrow+\infty} N_{2}(t) \leqslant\left(\frac{r_{2}^{u} \alpha_{2}^{u}}{r_{2}^{l}}\right)^{\frac{1}{\delta_{2}}} \exp \left\{r_{2}^{u} \alpha_{2}^{u} \tau\right\} \stackrel{\text { def }}{=} M_{2}
$$

For any small positive constant $\varepsilon>0$, from (2.4)-(2.5) it follows that there exists a $T_{1}>0$ such that for all $t>T_{1}$ and $i=1,2$,

$$
N_{i}(t)<M_{i}+\varepsilon
$$

For $t \geqslant T_{1}+\tau$, from (2.6) and the first equation of system (1.1), we have

$$
\begin{aligned}
\frac{d N_{1}(t)}{d t} & =r_{1}(t) N_{1}(t)\left[\frac{K_{1}(t)+\alpha_{1}(t) N_{2}^{\beta_{1}}\left(t-\tau_{2}(t)\right)}{1+N_{2}^{\beta_{1}}\left(t-\tau_{2}(t)\right)}-N_{1}^{\delta_{1}}\left(t-\sigma_{1}(t)\right)\right] \\
& \geqslant r_{1}(t) N_{1}(t)\left[\frac{K_{1}(t)+K_{1}(t) N_{2}^{\beta_{1}}\left(t-\tau_{2}(t)\right)}{1+N_{2}^{\beta_{1}}\left(t-\tau_{2}(t)\right)}-N_{1}^{\delta_{1}}\left(t-\sigma_{1}(t)\right)\right] \\
& =r_{1}(t) N_{1}(t)\left[K_{1}(t)-N_{1}^{\delta_{1}}\left(t-\sigma_{1}(t)\right)\right] \\
& \geqslant N_{1}(t)\left[r_{1}^{l} K_{1}^{l}-r_{1}^{u}\left(M_{1}+\varepsilon\right)^{\delta_{1}}\right] .
\end{aligned}
$$

Noting that

$$
\begin{aligned}
r_{1}^{\mathrm{l}} K_{1}^{\mathrm{l}}-\mathrm{r}_{1}^{\mathrm{u}}\left(M_{1}+\varepsilon\right)^{\delta_{1}} & \leqslant \mathrm{r}_{1}^{\mathrm{u}} K_{1}^{\mathrm{l}}-\mathrm{r}_{1}^{\mathrm{u}}\left(M_{1}+\varepsilon\right)^{\delta_{1}} \\
& \leqslant r_{1}^{\mathrm{u}}\left(K_{1}^{\mathrm{l}}-\left(M_{1}+\varepsilon\right)^{\delta_{1}}\right) \\
& \leqslant r_{1}^{\mathrm{u}}\left(\mathrm{K}_{1}^{\mathrm{l}}-\left(M_{1}\right)^{\delta_{1}}\right) \\
& \leqslant r_{1}^{\mathrm{u}}\left(\mathrm{K}_{1}^{\mathrm{l}}-\frac{\mathrm{r}_{1}^{\mathrm{u}} \alpha_{1}^{\mathrm{u}}}{\mathrm{r}_{1}^{\mathrm{l}}} \exp \left\{\delta_{1} r_{1}^{\mathrm{u}} \alpha_{1}^{\mathrm{u}} \tau\right\}\right) \\
& \leqslant \mathrm{r}_{1}^{\mathrm{u}}\left(\mathrm{K}_{1}^{\mathrm{l}}-\alpha_{1}^{\mathrm{u}}\right) \leqslant 0 .
\end{aligned}
$$

Integrating both sides of (2.7) from $t-\sigma_{1}(t)$ to $t$ leads to

$$
\ln \frac{N_{1}(t)}{N_{1}\left(t-\sigma_{1}(t)\right)} \geqslant \int_{t-\sigma_{1}(t)}^{t}\left[r_{1}^{l} K_{1}^{l}-r_{1}^{u}\left(M_{1}+\varepsilon\right)^{\delta_{1}}\right] d s \geqslant\left[r_{1}^{l} K_{1}^{l}-r_{1}^{u}\left(M_{1}+\varepsilon\right)^{\delta_{1}}\right] \tau,
$$

and so

$$
N_{1}\left(t-\sigma_{1}(t)\right) \leqslant N_{1}(t) \exp \left\{-\left[r_{1}^{l} K_{1}^{l}-r_{1}^{u}\left(M_{1}+\varepsilon\right)^{\delta_{1}}\right] \tau\right\}
$$


Substituting (2.9) into the first equation of system (1.1), using (2.9), for $t \geqslant T_{1}+\tau$, it follows that

$$
\begin{aligned}
\frac{d N_{1}(t)}{d t} & \geqslant r_{1}(t) N_{1}(t)\left[\frac{K_{1}(t)+K_{1}(t) N_{2}^{\beta_{1}}\left(t-\tau_{2}(t)\right)}{1+N_{2}^{\beta_{1}}\left(t-\tau_{2}(t)\right)}-N_{1}^{\delta_{1}}\left(t-\sigma_{1}(t)\right)\right] \\
& =r_{1}(t) N_{1}(t)\left[K_{1}(t)-N_{1}^{\delta_{1}}\left(t-\sigma_{1}(t)\right)\right] \\
& \geqslant N_{1}(t)\left[r_{1}^{l} K_{1}^{l}-r_{1}^{u} N_{1}^{\delta_{1}}\left(t-\sigma_{1}(t)\right)\right] \\
& \geqslant N_{1}(t)\left[r_{1}^{l} K_{1}^{l}-r_{1}^{u} N_{1}^{\delta_{1}}(t) \exp \left\{-\left[r_{1}^{l} K_{1}^{l}-r_{1}^{u}\left(M_{1}+\varepsilon\right)^{\delta_{1}}\right] \delta_{1} \tau\right\}\right],
\end{aligned}
$$

thus, as a direct corollary of Lemma 2.1, according to (2.10), one has

$$
\begin{aligned}
\liminf _{t \rightarrow+\infty} N_{1}(t) & \geqslant\left(\frac{r_{1}^{l} K_{1}^{l}}{r_{1}^{\mathfrak{u}}} \exp \left\{\left[r_{1}^{l} K_{1}^{l}-r_{1}^{\mathfrak{u}}\left(M_{1}+\varepsilon\right)^{\delta_{1}}\right] \delta_{1} \tau\right\}\right)^{\frac{1}{\delta_{1}}} \\
& =\left(\frac{r_{1}^{l} K_{1}^{l}}{r_{1}^{\mathfrak{u}}}\right)^{\frac{1}{\delta_{1}}} \exp \left\{\left[r_{1}^{l} K_{1}^{l}-r_{1}^{\mathfrak{u}}\left(M_{1}+\varepsilon\right)^{\delta_{1}}\right] \tau\right\} .
\end{aligned}
$$

Setting $\varepsilon \rightarrow 0$, it follows that

$$
\liminf _{t \rightarrow+\infty} N_{1}(t) \geqslant \frac{1}{2}\left(\frac{r_{1}^{l} K_{1}^{l}}{r_{1}^{u}}\right)^{\frac{1}{\delta_{1}}} \exp \left\{\left[r_{1}^{l} K_{1}^{l}-r_{1}^{u}\left(M_{1}\right)^{\delta_{1}}\right] \tau\right\} \stackrel{\text { def }}{=} m_{1} .
$$

Similarly to the analysis of (2.7)-(2.12), by applying (2.6), from the second equation of system (1.1), we can also have

$$
\liminf _{t \rightarrow+\infty} N_{2}(t) \geqslant \frac{1}{2}\left(\frac{r_{2}^{l} K_{2}^{l}}{r_{2}^{u}}\right)^{\frac{1}{\delta_{2}}} \exp \left\{\left[r_{2}^{l} K_{2}^{l}-r_{2}^{u}\left(M_{2}\right)^{\delta_{2}}\right] \tau\right\} \stackrel{\text { def }}{=} m_{2} .
$$

(2.4)-(2.5) and (2.12)-(2.13) show that under the assumptions of Theorem 1.1, system (1.1) is permanent. This ends the proof of Theorem 1.1.

\section{Numeric simulations}

In this section we will give an example to show the feasibility of the Theorem 1.1.

\section{Example 3.1.}

$$
\begin{aligned}
\frac{d N(t)}{d t} & =N_{1}(t)\left[\frac{2+\left(4-\frac{1}{2} \cos (t)\right) N_{2}^{2}(t)}{1+N_{2}^{2}(t)}-N_{1}^{\frac{1}{2}}(t)\right] \\
\frac{d N_{2}(t)}{d t} & =N_{2}(t)\left[\frac{1+\left(3+\frac{1}{10} \sin (t)\right) N_{1}^{2}(t)}{1+N_{1}^{2}(t)}-N_{2}^{\frac{1}{2}}(t)\right] .
\end{aligned}
$$

Corresponding to system (1.1), one has

$$
r_{1}(t)=r_{2}(t)=1, \quad \alpha_{1}(t)=4-\frac{1}{2} \cos (t), \quad \alpha_{2}(t)=3+\frac{1}{10} \sin (t), K_{1}(t)=2, K_{2}(t)=1 .
$$

Obviously, $\alpha_{i}(t)>K_{i}(t), i=1,2$, hence, the conditions of Theorem 1.1 hold, it follows from Theorem 1.1 that system (3.1) is permanent. Figs. 1 and 2 also support this assertion. 


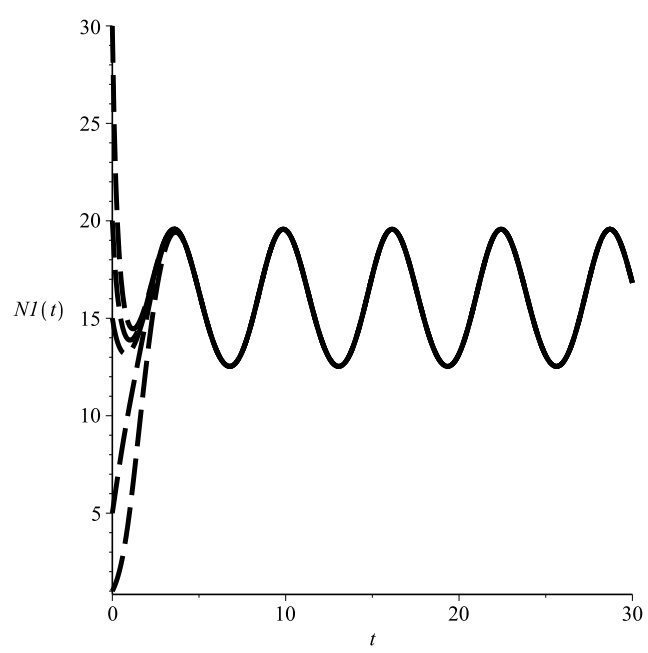

Figure 1: Dynamic behavior of the first species in system (3.1) with the initial conditions $\left(\mathrm{N}_{1}(0), \mathrm{N}_{2}(0)\right)=(5,5)$, $(1,1),(15,13),(30,15)$, and $(20,20)$, respectively.

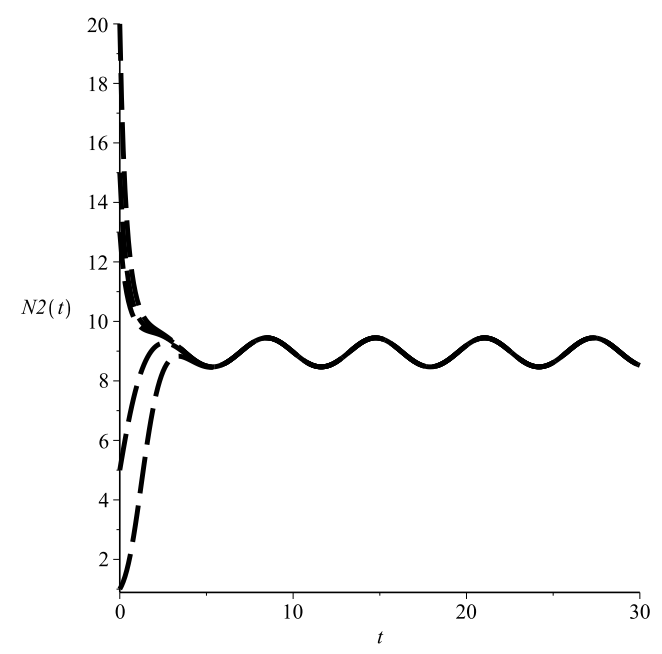

Figure 2: Dynamic behavior of the second species in system (3.1) with the initial conditions $\left(\mathrm{N}_{1}(0), \mathrm{N}_{2}(0)\right)=$ $(5,5),(1,1),(15,13),(30,15)$, and $(20,20)$, respectively.

\section{Discussion}

Li [21] proposed a model of mutualism (i.e., system (1.3)). Under the assumption $\alpha_{i}>K_{i}, i=1,2$, by using the coincidence degree theory, they showed that the system admits at least one positive periodic solution. In this paper, we generalize the system (1.3) to the nonlinear case. By using the theory of differential inequality, and applying the analysis technique of Chen [2], we also obtain a set of sufficient conditions which ensure the permanence of the system.

Numeric simulations (Example 3.1) supports our finding. At the end of the paper, we would like to mention that from Figs. 1 and 2, one could see that system (3.1) admits unique positive periodic solution which is globally attractive, hence, it seems interesting to investigate the stability property of the system (1.1), we leave this for future investigation.

\section{Acknowledgment}

The research was supported by the Natural Science Foundation of Fujian Province (2019J01783).

\section{References}

[1] D. H. Boucher, The biology of mutualism: ecology and evolution, Croom Helm, London, (1985). 1, 1

[2] F. D. Chen, On a nonlinear non-autonomous predator-prey model with diffusion and distributed delay, J. Comput. Appl. Math., 180 (2005), 33-49. 1, 4

[3] B. G. Chen, The influence of commensalism on a Lotka-Volterra commensal symbiosis model with Michaelis-Menten type harvesting, Adv. Difference Equ., 2019 (2019), 14 pages. 1

[4] L. J. Chen, L. J. Chen, Z. Li, Permanence of a delayed discrete mutualism model with feedback controls, Math. Comput. Modelling, 50 (2009), 1083-1089. 1

[5] J. H. Chen, R. X. Wu, A commensal symbiosis model with non-monotonic functional response, Comm. Math. Biol. Neur., 2017 (2017), 8 pages. 1

[6] F. D. Chen, H. L. Wu, X. D. Xie, Global attractivity of a discrete cooperative system incorporating harvesting, Adv. Difference Equ., 2016 (2016), 12 pages. 1

[7] L. J. Chen, X. D. Xie, Permanence of an n-species cooperation system with continuous time delays and feedback controls, Nonlinear Anal. Real World Appl., 12 (2001), 34-38. 1

[8] L. J. Chen, X. D. Xie, L. J. Chen, Feedback control variables have no influence on the permanence of a discrete N-species cooperation system, Discrete Dyn. Nat. Soc., 2009 (2009), 10 pages. 1

[9] F. D. Chen, X. D. Xie, X. F. Chen, Dynamic behaviors of a stage-structured cooperation model, Comm. Math. Biol. Neur., 2015 (2015), 19 pages. 1 
[10] F. D. Chen, X. D. Xie, Z. S. Miao, L. Q. Pu, Extinction in two species nonautonomous nonlinear competitive system, Appl. Math. Comput., 274 (2016), 119-124. 1, 2.1

[11] F. D. Chen, Y. L. Xue, Q. F. Lin, X. D. Xie, Dynamic behaviors of a Lotka-Volterra commensal symbiosis model with density dependent birth rate, Adv. Difference Equ., 2018 (2018), 14 pages. 1

[12] F. D. Chen, J. H. Yang, L. J. Chen, X. D. Xie, On a mutualism model with feedback controls, Appl. Math. Comput., 214 (2009), 581-587. 1

[13] A. M. Dean, A simple model of mutualism, Amer. Natural., 121 (1983), 409-417. 1, 1

[14] K. Gopalsamy, Stability and Oscillations in Delay Differential Equations of Population Dynamics, Kluwer Academic Publishers, Dordrecht, (1992). 1, 1

[15] X. Y. Guan, F. D. Chen, Dynamical analysis of a two species amensalism model with Beddington-DeAngelis functional response and Allee effect on the second species, Nonlinear Anal. Real World Appl., 48 (2019), 71-93. 1

[16] R. Y. Han, X. D. Xie, F. D. Chen, Permanence and global attractivity of a discrete pollination mutualism in plant-pollinator system with feedback controls, Adv. Difference Equ., 2016 (2016), 17 pages. 1

[17] M. X. He, F. D. Chen, Extinction and stability of an impulsive system with pure delays, Appl. Math. Lett., 91 (2019), $128-136$.

[18] M. X. He, Z. Li, F. D. Chen, Permanence, extinction and global attractivity of the periodic Gilpin-Ayala competition system with impulses, Nonlinear Anal. Real World Appl., 11 (2010), 1537-1551. 1

[19] C. Q. Lei, Dynamic behaviors of a non-selective harvesting May cooperative system incorporating partial closure for the populations, Comm. Math. Biol. Neur., 2018 (2018), 23 pages. 1

[20] C. Q. Lei, Dynamic behaviors of a stage-structured commensalism system, Adv. Difference Equ., 2018 (2018), 20 pages.

[21] Y. K. Li, On a periodic mutualism model, ANZIAM J., 42 (2001), 569-580. 1

[22] Z. Li, F. D. Chen, Extinction and almost periodic solutions of a discrete Gilpin-Ayala type population model, J. Difference Equ. Appl., 19 (2013), 719-737. 1, 1, 4 1

[23] T. X. Li, Y. V. Rogovchenko, Oscillation criteria for second-order superlinear Emden-Fowler neutral differential equations, Monatsh. Math., 184 (2017), 489-500. 1

[24] Y. K. Li, G. T. Xu, Positive periodic solutions for an integrodifferential model of mutualism, Appl. Math. Lett., 14 (2001), 525-530. 1, 1

[25] Q. X. Lin, X. D. Xie, F. D. Chen, Q. F. Lin, Dynamical analysis of a logistic model with impulsive Holling type-II harvesting, Adv. Difference Equ., 2018 (2018), 22 pages.

[26] Z. S. Miao, X. D. Xie, L. Q. Pu, Dynamic behaviors of a periodic Lotka-Volterra commensal symbiosis model with impulsive, Comm. Math. Biol. Neur., 2015 (2015), 15 pages.

[27] L. Q. Pu, X. D. Xie, F. D. Chen, Z. S. Miao, Extinction in two-species nonlinear discrete competitive system, Discrete Dyn. Nat. Soc., 2016 (2016), 10 pages. 1

[28] C. L. Wolin, L. R. Lawlor, Models of facultative mutualism: density effects, Amer. Natural., 144 (1984), 843-862. 1

[29] R. X. Wu, Dynamic behaviors of a nonlinear amensalism model, Adv. Difference Equ., 2018 (2018), 13 pages.

[30] R. X. Wu, L. Li, Q. F. Lin, A Holling type commensal symbiosis model involving Allee effect, Comm. Math. Biol. Neur., 2018 (2018), 13 pages.

[31] X. D. Xie, F. D. Chen, Y. L. Xue, Note on the stability property of a cooperative system incorporating harvesting, Discrete Dyn. Nat. Soc., 2014 (2014), 5 pages. 1

[32] X. D. Xie, F. D. Chen, K. Yang, Y. L. Xue, Global attractivity of an integro-differential model of mutualism, Abstract Appl. Anal., 2014 (2014), 6 pages. 1

[33] X. D. Xie, Y. L. Xue, R. X. Wu, Global attractivity in a discrete mutualism model with infinite deviating arguments, Discrete Dyn. Nat. Soc., 2017 (2017), 8 pages. 1

[34] Y. L. Xue, X. D. Xie, F. D. Chen, R. Y. Han, Almost periodic solution of a discrete commensalism system, Discrete Dyn. Nat. Soc., 2015 (2015), 11 pages. 1

[35] K. Yang, Z. S. Miao, F. D. Chen, X. D. Xie, Influence of single feedback control variable on an autonomous Holling-II type cooperative system, J. Math. Anal. Appl., 435 (2016), 874-888.

[36] K. Yang, X. D. Xie, F. D. Chen, Global stability of a discrete mutualism model, Abstr. Appl. Anal., 2014 (2014), 7 pages. 1

[37] L. Y. Yang, X. D. Xie, F. D. Chen, Dynamic behaviors of a discrete periodic predator-prey-mutualist system, Discrete Dyn. Nat. Soc., 2015 (2015), 11 pages. 1, 1 